\title{
Disposição final dos resíduos sólidos urbanos: diagnóstico da gestão do município de Santo Antônio de Goiás
}

\author{
Final disposal of municipal solid waste: diagnosis of management city of Santo Antônio de Goiás \\ Evaldo de Melo Ferreira, Karla Alcione da Silva Cruvinel, Eliabe Soares da Costa \\ 'Mestrando em Agronomia na Universidade Federal de Goiás (UFG) \\ ${ }^{2}$ Doutoranda em Ciências Ambientais. Professora Assistente da Universidade Federal de Goiás (UFG) \\ ${ }^{3}$ Agrônomo pelo Centro Universitário de Goiás
}

\begin{abstract}
Resumo
A gestão dos resíduos sólidos é a parte que envolve a política relacionada a esses. O gerenciamento são as ferramentas, a parte prática. No ano de 2010, o Brasil avançou na temática relacionada à gestão pública de resíduos sólidos urbanos e industriais. Foi instituída, por exemplo, a responsabilidade sobre o material gerado. Com a Lei de $\mathrm{N}^{\circ} 12$. 305, o gerador tornou-se responsável pelo que é descartado. De acordo com essa legislação, na gestão e gerenciamento de resíduos sólidos, deve ser observada a seguinte ordem de prioridade: não geração, redução, reutilização, reciclagem, tratamento dos resíduos sólidos e disposição final ambientalmente adequada dos rejeitos. O presente trabalho foi desenvolvido no município de Santo Antônio de Goiás, GO, Brasil, com o objetivo de avaliar a área de disposição final dos resíduos do município. No levantamento da qualidade da área utilizada para disposição final dos resíduos sólidos, foi utilizado o método Índice de Qualidade de Aterro de Resíduos (IQR) preconizado pela Companhia Ambiental do Estado de São Paulo (CETESB, 2011). Com o resultado pode-se concluir que a área está inadequada, obtendo nota igual a 3,7 .
\end{abstract}

Palavras-chaves: Vazadouros, resíduos urbanos, Índice de Qualidade de Aterro de Resíduos (IQR).

\begin{abstract}
The solid waste management is the part that involves related to such policy. The management are the tools, the practical part. In 2010, Brazil has advanced in the subject related to public management of urban and industrial solid waste. For example, it established the responsibility for the material generated. With the Law $\mathrm{N}^{\circ} 12.305$, the generator has become responsible for what is discarded. According to this legislation, management and solid waste management is to be observed the following order of priority: no generation, reduction, reuse, recycling, solid waste treatment and environmentally sound disposal of waste. This work was developed in Santo Antônio de Goiás, GO, Brazil, with the objective of evaluating the area of final waste disposal of the municipality. In the survey of the quality of the area used for disposal of solid waste, the Índice de Qualidade de Aterro de Resíduos (IQR) method recommended by the Companhia Ambiental do Estado de São Paulo (CETESB, 2011) was used. With this result we can conclude that the area is inadequate, resulting grade equal to 3,7 .
\end{abstract}

Keywords:Municipal landfill, waste solid urban, Quality Index Landfill Waste (QILW). 


\section{INTRODUÇÃO}

Os resíduos sólidos gerados nas cidades brasileiras e em localidades de diferentes países, ainda são um problema. Em países ricos que geram maiores quantidades de resíduos, existe mais capacidade de equacionamento da gestão, por um somatório de fatores que incluem recursos econômicos, preocupação ambiental da população e desenvolvimento tecnológico (JACOBI \& BESSEN, 2011).

Mesmo com a preocupação da comunidade internacional com a qualidade do meio ambiente, o que acontece na maioria das vezes é um desenvolvimento não sustentável na gestão dos resíduos sólidos municipais. Os métodos para a coleta, transporte e depósito dos resíduos sólidos não levam em conta as consequências que o mau uso e o tratamento inadequado deles podem acarretar no meio ambiente (SIMONETO \& LÖBLER, 2013).

No entanto, os resíduos sólidos possuem grande potencial econômico. Quando reciclados podem ser fonte de renda para populações carentes. No ano de 2010, a Lei de $\mathrm{N}^{\mathrm{o}} 12.305$, também conhecida como Política Nacional de Resíduos Sólidos (PNRS) trouxe diversas mudanças e junto a essas, ferramentas úteis para a gestão e gerenciamento do lixo produzido nas cidades.

Analisando toda a cadeia geradora de resíduos sólidos (fabricação do produto, venda, uso e descarte), a produção e o descarte final são os que exigem maior cuidado. No uso de matérias primas surgem os materiais provenientes do desperdício e das sobras do processo. Nessa fase e no descarte final, os aspectos ambientais passam a exigir maior cuidado com sua gestão ambiental. O solo, água, ar e a saúde humana, são alguns dos atingidos pela má gestão dos resíduos gerados. Nessa cadeia geradora, todos são responsáveis pelo que insere no meio, fabricante, consumidor e gestores públicos. Dai surge o conceito de logística reversa, que é um conjunto de ações, procedimentos e meios que viabilizam a coleta e a restituição dos resíduos sólidos ao setor empresarial (BRASIL, 2010).

Estudos que avaliem a forma de gestão das áreas de disposição dos resíduos sólidos consistem em uma demanda atual e de expressiva importância para o estabelecimento de medidas controle dessas áreas. Para a avaliação da qualidade da disposição final de resíduos, a Companhia Ambiental do Estado de São Paulo (CETESB) criou o Índice de Qualidade de Aterro de Resíduos (IQR). Esse método passou por transformações ao longo dos anos. De acordo com a CETESB (2011, p. 1), o IQR: "avalia as características locacionais, estruturais e operacionais dos locais de tratamento e disposição de resíduos".

Tendo como foco principal nesse trabalho o tema acima discutido, optou-se por trabalhar com o município de Santo Antônio de Goiás. Sua população está em torno de 4.703 habitantes, tendo uma área igual a 132,805 km² (IBGE, 2013). O IQR foi uma das ferramentas utilizadas no diagnóstico da gestão da área de disposição final dos resíduos urbanos de Santo Antônio de Goiás, sendo este o objetivo principal do presente trabalho.

\section{METODOLOGIA}

O método para a execução da pesquisa é constituído por cinco etapas diferentes:

- Primeira etapa: levantamento bibliográfico sobre o tema;

- Segunda etapa: consulta a documentos oficiais da prefeitura e documentos online da Secretaria de Estado do Meio Ambiente e Recursos Hídricos de Goiás (SEMARH GO);

- Terceira etapa: visitas técnicas á área do lixão;

- Quarta etapa: comparação dos aspectos encontrados com as leis e normais aplicáveis;

- Quinta etapa: avaliação do IQR (CETESB, 2011).

Foram realizadas buscas em artigos científicos em periódicos nacionais e internacionais, e consultadas as normas da ABNT, além de publicações da SEMARH GO e dados do site do Instituto Brasileiro de Geografia e Estatística (IBGE). A NBR 8419 (ABNT, 1992), NBR 13896 (1997) e a Instrução Normativa 11 de 2013 da SEMARH-GO foram utilizadas como referência neste trabalho.

Para levantamento de dados dos resíduos sólidos gerados no município, foi consultado o Plano de Gerenciamento Integrado dos Resíduos Sólidos (PGRIS). Outros documentos da prefeitura e relatórios da SEMARH GO foram utilizados objetivando uma abordagem sobre os aspectos históricos, ambientais e sociais da área de estudo. 
As vistorias à área de disposição dos resíduos foram acompanhadas pela gestora ambiental da prefeitura que contribuiu com informações, no entanto, uma grande totalidade dos dados aqui apresentados foi baseada na inspeção visual do local, no qual se buscou conhecer e avaliar os aspectos relacionados a:

- Infra-estrutura existente;

- Condições de operação;

- Identificação dos resíduos dispostos na área;

- Possíveis impactos ambientais ao solo, ar e saúde humana;

- Presença de animais;

- Presença de catadores.

Os aspectos locacionais também foram avaliados e para isto buscou-se levantar a distancia da área com relação à cidade, a residências rurais e mananciais hídricos utilizando de vistorias in loco. Objetivou-se desse modo verificar a situação encontrada com as exigências estabelecidas na IN 011/2013 da SEMARH-GO, que dispõe sobre o licenciamento ambiental dos projetos de disposição final dos resíduos sólidos urbanos na modalidade Aterro Sanitário Simplificado, nos municípios de Goiás.

Para melhor visualização da área onde está localizada a disposição final dos resíduos sólidos de Santo Antônio de Goiás, em relação à área urbana e ao corpo hídrico mais próximo e ainda a tipologia de solo da área, foi confeccionado um mapa temático no Laboratório de Processamento de Imagens e Geoprocessamento da Universidade Federal de Goiás (LAPIG-UFG).

O software utilizado foi o Arcmap ${ }^{\circledR}$ versão 10.1. Entre algumas das vantagens desse programa computacional é que ao adicionar um arquivo apoio para o projeto de mapeamento, um crédito de camada de serviço para o mapa base (ou qualquer conjunto de dados transmitidos como um serviço de $\operatorname{ArcGIS}{ }^{\circledR}$ online) aparece automaticamente na exibição de layout (IFWIS, 2014). Também foram utilizadas as bases de dados do Sistema Estadual de Geoinformação de Goiás (SIEG, 2004) e do Instituto Brasileiro de Geografia e Estatística (IBGE).

Também as resoluções RDC 306 de 2004 da Agência Nacional de Vigilância Sanitária (ANVISA) e Conama 358 de 2005 e 307 de 2002, foram instrumentos de análise, utilizadas para a avaliação dos aspectos legais da disposição final dos resíduos de serviço de saúde (RSS) e resíduos da construção civil (RCC) gerados no município.

De posse dos dados obtidos, estes foram avaliados com base nos documentos legais e normativos pertinentes para avaliação das condições observadas in loco, caracterizando assim a quarta etapa do trabalho.

Considerando ainda a necessidade de avaliação mais fundamentada, buscou-se, como ultima etapa, adotar o IQR para avaliar o sistema de disposição final de resíduos do município de Santo Antônio de Goiás. O método classifica os locais de destinação dos resíduos, processada a partir da aplicação de um questionário respondido através da vistoria. Os quesitos analisados se baseiam nas características locacionais, estruturais e operacionais. Todas as atividades de concepção metodológica, levantamentos de dados e sistematização de informações foram adotados conforme proposta apresentada pela CETESB (2011).

Quadro 1. Enquadramento da área de disposição de resíduos.

\begin{tabular}{|c|c|}
\hline IQR & AVALIAÇÃO \\
\hline 0 a 6,0 & Condições inadequadas \\
\hline 6,1 a 8,0 & Condições controladas \\
\hline 8,1 a 10,0 & Condições adequadas \\
\hline
\end{tabular}

Fonte: Adaptado de CESTEB (2011).

Com o auxílio do IQR, o Estado de São Paulo desde 2007 tem organizado e disponibilizado anualmente as informações sobre as condições ambientais e sanitárias dos locais de destinação final de resíduos domiciliares nos municípios paulistas. Isso é utilizado na elaboração do Inventário Estadual de Resíduos Domiciliares e o aprimoramento dos mecanismos de gestão ambiental (CETESB, 2013). 


\section{RESULTADOS E DISCUSSÃO}

De responsabilidade da Prefeitura Municipal de Santo Antônio de Goiás, a área de disposição de resíduos do município está localizada sob as coordenadas geográficas (em graus decimais) Latitude S $16^{\circ} 28^{\prime} 30.22^{\prime \prime}$ e Longitude W 49¹9'48.43". Quando o trabalho foi desenvolvido, essa não possuía licença ambiental de funcionamento, mas já foi operado como aterro controlado e licenciado pelo órgão ambiental nesta modalidade.

Em fevereiro/março de 2014 as características de aterro controlado não existiam, recebendo aproximadamente três toneladas de resíduos sólidos urbanos diariamente. Segundo Goiás (2011, p. s/n): "a área está sendo utilizada para disposição final dos resíduos sólidos urbanos a mais de 8 anos". A coleta dos resíduos sólidos urbanos na cidade é realizada diariamente, tendo rotas divididas que atendem cada região da cidade duas vezes por semana.

A municipalidade de Santo Antônio de Goiás está em processo de estabelecimento de consórcio público para a construção de aterro sanitário com a participação Santo Antônio de Goiás, Inhumas, Brazabrantes, Goianira e Nova Veneza. Os consórcios entre municípios para estabelecimento de local para disposição final dos resíduos sólidos urbanos tem sido uma pratica entre municípios de outros estados brasileiros e é incentivada pela PNRS. Alguns exemplos de consórcios para construção de aterros são os realizados em Alto Sapucaí e Zona da Mata, ambos em Minas Gerais (ABES MG, 2014), um dos estados pioneiros neste processo e com vários consórcios formados atualmente.

O Plano Gerenciamento Integrado de Resíduos Sólidos (PGIRS) do município foi elaborado no ano de 2013. Apesar de ser um documento obrigatório para os municípios, conforme a PNRS, a elaboração dele para o município estudado só ocorreu após o prazo estabelecido no artigo 55 da lei, que era até 2 de agosto de 2012 . No entanto, é esta a realidade de aproximadamente $70 \%$ dos municípios brasileiros, conforme informa a Confederação Nacional dos Municípios (CNM, 2013).

A consulta ao PGIRS do município estudado propiciou a identificação de aspectos importantes para caracterizar os resíduos do município, como a geração per capita de resíduos sólidos e a composição gravimétrica. A geração per capita é igual a $0,827 \mathrm{~kg} /$ hab.dia, conforme apresentado na Tabela 1.

Tabela 1. Resíduos gerados por hab/dia.

\begin{tabular}{lc}
\hline Geração Per capita de Resíduos Sólidos em Santo Antônio-GO \\
\hline Resíduo gerado por dia & $0,83 \mathrm{~kg} / \mathrm{hab}$.dia \\
Resíduo gerado por mês & $24 \mathrm{~kg} / \mathrm{hab} . \mathrm{mês}$ \\
Resíduo gerado por ano & $297 \mathrm{~kg} / \mathrm{hab}$.ano \\
\hline
\end{tabular}

Fonte: Adaptado de Santo Antônio de Goiás (2013).

A composição gravimétrica apresentada no PGIRS demonstrou uma porcentagem representativa de resíduos como tecido (29\%), sendo o resíduo mais gerado, situação atípica para a maioria dos municípios brasileiros (Figura 1). O resíduo mais presente nos resíduos sólidos urbanos coletados no Brasil é a matéria orgânica, com mais de $51 \%$ de representatividade sobre a massa total dos resíduos gerados (ABRELPE, 2012). Segundo a Gestora Ambiental isso pode ser explicado pela existência de uma confecção de roupas em Santo Antônio de Goiás.

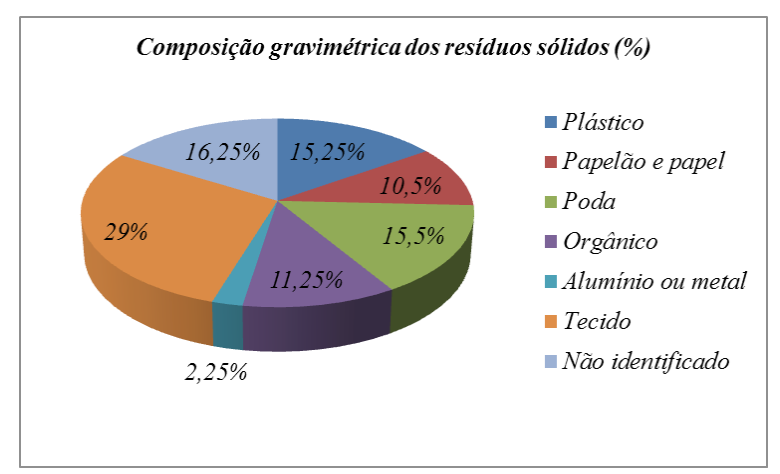

Figura 1 - Composição gravimétrica dos resíduos sólidos.

Fonte: Adaptado de Santo Antônio de Goiás (2013). 


\section{1. Aspectos operacionais da área de disposição dos resíduos}

Em Goiás não se trabalha com a modalidade de aterro controlado desde o ano de 2010, já que o órgão ambiental (SEMARH-GO) adotou a metodologia de Aterro Sanitário Simplificado, conforme a IN 011/2013. Para a área de disposição final dos resíduos municipais ser licenciada nessa modalidade, o município tem que possuir população até 100 mil habitantes e o seu aterro ter uma estrutura mínima exigida, como impermeabilização da trincheira, acesso e isolamento do local, sistema de drenagem de águas pluviais, sistemas de drenagem e remoção de percolados, sistema de tratamento de percolados, drenagem e tratamento dos gases e sistema de controle ambiental (GOIÁS, 2013).

Segundo a NBR 13896 (ABNT, 1997), para ser construído um aterro sanitário, é necessário atender a determinados requisitos. O local deve permitir a minimização do impacto ambiental causado por sua instalação, a aceitação da população deve ser maximizada, o local deve estar de acordo com o zoneamento da região, possibilidade de utilização por um longo tempo, necessitando apenas de mínimo de obras para início das operações (ABNT, 1997).

Embora ele já tenha sido licenciamento como aterro controlado, ele não possui estrutura para ser licenciado como aterro sanitário simplificado. Os resíduos são depositados em área sem impermeabilização e cobertos com terra três vezes por semana (Figura 2). A ausência da cobertura diária dos resíduos possibilita a presença considerável de animais, como urubus e cães.

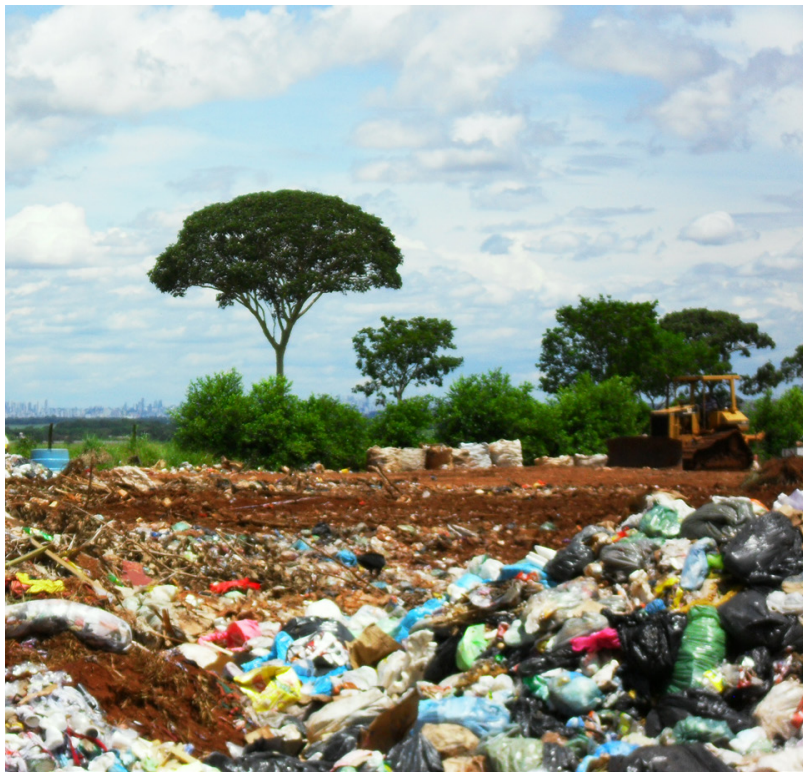

Figura 2 - Área onde o lixo é depositado.

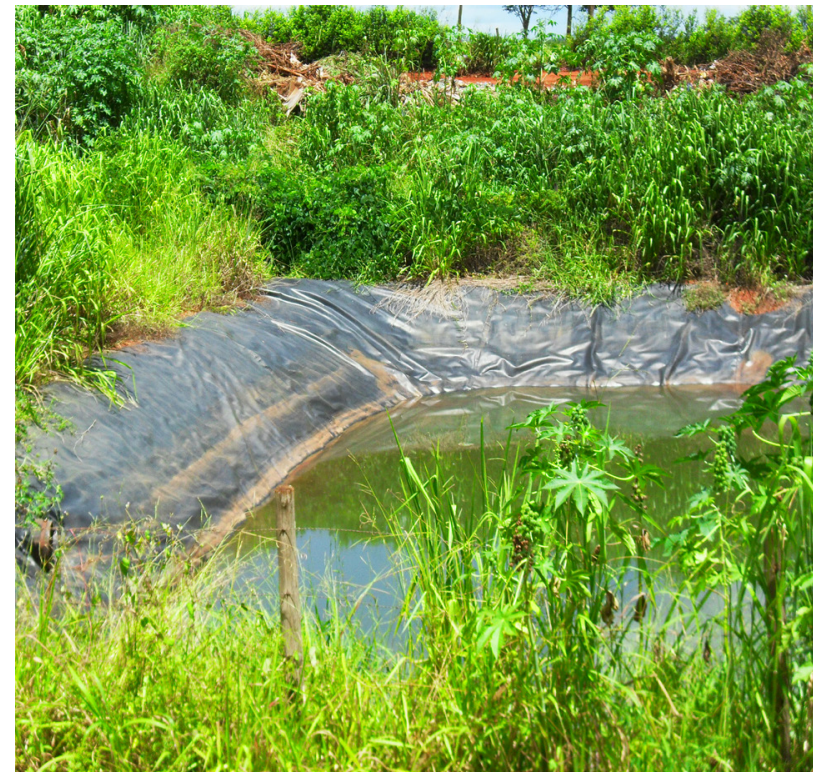

Figura 3 - Lagoa para recebimento do percolado.

Foi identificada a existência de uma lagoa para recebimento do percolado (Figura 3), que não está em uso devido à ausência de dreno de percolado e com a manta apresentando problema de instalação. O que indica a infiltração do percolado gerado, já que não existe uma drenagem desses. Uma nova vala está sendo aberta no local, no entanto, sem critérios técnicos de Engenharia. A área possui cerca e a presença de cinturão verde em parte dela, o que não impede a presença de animais e catadores.

Esses catadores são presença constante no local, aproximadamente quatro catadores de materiais recicláveis trabalham dentro da área. Esses são responsáveis pela segregação do material com valor comercial que é encaminhado para cooperativa acompanhada pela incubadora de empresas da Universidade Federal de Goiás (UFG).

A atividade de catação, apesar de ser proibida, é realidade em vários municípios brasileiros. De acordo com o Ministério do Meio Ambiente (2014, p. s/n), "No Brasil, a estimativa é de que existam 600 mil catadores de materiais recicláveis [...]".

De maneira geral, o local não atende aos requisitos mínimos para funcionamento na modalidade de aterro sanitário. A estrutura mínima necessária contemplada tanto na IN 011/2013 (GOIÁS, 2013), na NBR 8419 (ABNT, 1992) e NBR 13896 (ABNT, 1997) não foram identificadas no local. 


\section{2. Disposição final dos resíduos especiais gerados no município}

Além dos resíduos domésticos outros, como resíduos da construção, resíduos de serviço de saúde, pneus, eletroeletrônicos e industriais são gerados nas cidades e parcialmente encaminhados para o aterro do município. Esses resíduos também foram estudados neste trabalho e procurou-se identificar a forma de destinação final adotada pelo município para eles.

Quanto à presença de RCC, ressalta-se que eles não foram identificados na área de disposição de resíduos. Segundo a Gestora Ambiental de Santo Antônio, os RCC são coletados uma vez por semana nas residências e utilizados na pré-pavimentação de estradas vicinais. Considerando a classificação apresentada pela CONAMA 307 de 2002, alterada pela CONAMA 431 de 2011, os resíduos utilizados na pré-pavimentação citada acima são os pertencentes à Classe A. Não podendo ser encaminhados para aterros sanitários ou áreas de bota-fora.

Apesar de ter sido identificada essa forma de reaproveitamento dos RCC, não foi verificada uma forma real e segura de reciclagem dos RCC, processo que deveria ser priorizado. Essa é uma alternativa para economizar os recursos naturais utilizados como matéria-prima na construção civil, além de uma possibilidade de redução nos custos de construção e do volume final dos resíduos a serem dispostos (LIMA \& CABRAL, 2013).

Com relação aos RSS, consta no PGIRS que eles são encaminhados para a área de disposição de resíduos do município como forma de destinação final. No entanto, não foram identificados vestígios de disposição dos RSS no local e, segundo a Gestora Ambiental, eles tem sido encaminhado para o processo de incineração realizado pela empresa terceirizada. Sendo a coleta e destinação final realizada também por essa empresa.

A Resolução Conama No 358 de 29 de abril de 2005, assim como a RDC 306 de 2004, trabalham com divisão desses resíduos em grupos, proporcionando desta forma a melhor gestão. Essas resoluções indicam como responsabilidade pelo gerenciamento destes resíduos, etapas que incluem desde a geração até a disposição final, os geradores, não cabendo à prefeitura realizar coleta e nem arcar com a destinação final em órgãos privados.

Pneumáticos e eletrônicos são parcialmente encaminhados para o aterro. Os pneus são recolhidos pela empresa contratada e os eletrônicos são encaminhados para uma escola estadual onde funciona um ponto de coleta de materiais eletrônicos que recebe: pilhas, baterias, peças e o que é considerado como inservível pela população. Entretanto, no dia de uma das visitas técnicas foi possível verificar a presença de resíduos eletrônicos e pneus na área. O processo de destinação final dos resíduos gerados no município segue processos diferenciados.

\section{3. Aspectos locacionais}

Com relação aos aspectos locacionais da área foram observados os requisitos presentes na IN de número 11/2013 da SEMARH/GO. A instrução estabelece distâncias mínimas entre a área de disposição dos resíduos e outros pontos.

$\mathrm{O}$ aterro deve ficar distante $3,0 \mathrm{Km}$ de centros urbanos. De acordo com o PGIRS, o lixo é descartado na zona rural a leste da cidade, distante aproximadamente 3,0 $\mathrm{Km}$ do centro de Santo Antônio de Goiás, ao analisar a distâncias no Google Earth ${ }^{\circledR}$, com a imagem datada de 17 de junho de 2010, verificou-se uma distancia menor da informada anteriormente, sendo 2,0 Km, o que não atende a IN $011 / 2013$.

A distância estabelecida para imóveis rurais, necessária ao licenciamento da área na IN é de 500 metros. A inspeção no local e levantamento das imagens no Google Earth ${ }^{\circledR}$ permitiram identificar três residências rurais nas proximidades, sendo a mais próxima localizada a 780 metros.

Relativo aos mananciais hídricos deve-se considerar a distância mínima de $300 \mathrm{~m}$. Esse aspecto também foi avaliado e verificou-se que o manancial mais próximo à área é um lago localizado a aproximadamente 900 metros. O córrego mais próximo á área é o Córrego do Meio e está a 1.200 metros do local estudado.

Quando a área definida estiver à montante da captação de abastecimento público deverá manter uma distância mínima de 2.500 metros desse ponto e afastamento de 500 metros do corpo hídrico. Segundo dados do PGIRS o abastecimento é feito por meio de poços artesianos, onde a Empresa de Saneamento de Goiás (SANEAGO) realiza a aplicação de desinfetantes, sendo assim, não é necessária a avaliação desse parâmetro. 
Para área localizada na zona de amortecimento de Unidade de Conservação (Ucs), deve-se obter anuência do órgão gestor da referida unidade, conforme previsto na resolução CONAMA ${ }^{\circ}$ 428/2010 ou sua atualização. Não existem UCs nas proximidades da área.

A declividade da área foi identificada visualmente no momento da vistoria e por meio do Google Earth ${ }^{\circledR}$ pode-se afirmar que o terreno possui pequena declividade, não superando o preconizado na IN $011 / 2013$, declividade menor que $20 \%$. A aplicação de maquinário pesado na operação do aterro é garantido nessa faixa de declividade, o que consiste em um indicador da aptidão para aterro sanitário da área estudada.

Levantando as dimensões da área, conseguiu-se identificar a área total do local e o espaço já utilizado. O local possui $26.600 \mathrm{~m}^{2}$ de área total e aproximadamente $77 \%$ da área total já foi utilizada para disposição de resíduos sólidos urbanos, sobrando apenas $5.900 \mathrm{~m}^{2}$. Um dos requisitos para instalação do aterro sanitário simplificado é a necessidade de área para atender a um alcance de projeto de 15 anos. Portanto, dentro desse aspecto, este local não poderia ser licenciado. O que poderia ser solucionado com a aquisição de uma nova área contigua a atual.

O mapa produzido (Figura 4) permitiu identificar, entre outros aspectos, o tipo de solo. De forma geral a área está sobre solos do tipo Latossolos, sendo esses predominantes em área de Cerrado. Segundo a Agência de Informação Embrapa (2007, p. s/n): "apresentam teor de silte inferior a 20\% e argila variando entre 15\% e 80\%.". De acordo com o Sistema Brasileiro de Classificação de Solos-SBCS (2006, p. 152): "esses tem composição areno-quartizosa e são solos profundos e de um modo geral, os teores da fração argila no solum aumentam gradativamente com a profundidade, ou permanecem constantes ao longo do perfil." Desta forma, a presença de argila e características de solos profundos, o caracteriza como um solo mais resistente e com baixa percolação, apresentando barreiras para a contaminação do lençol freático.

\section{Localização do lixão de Santo Antônio de Goiás}

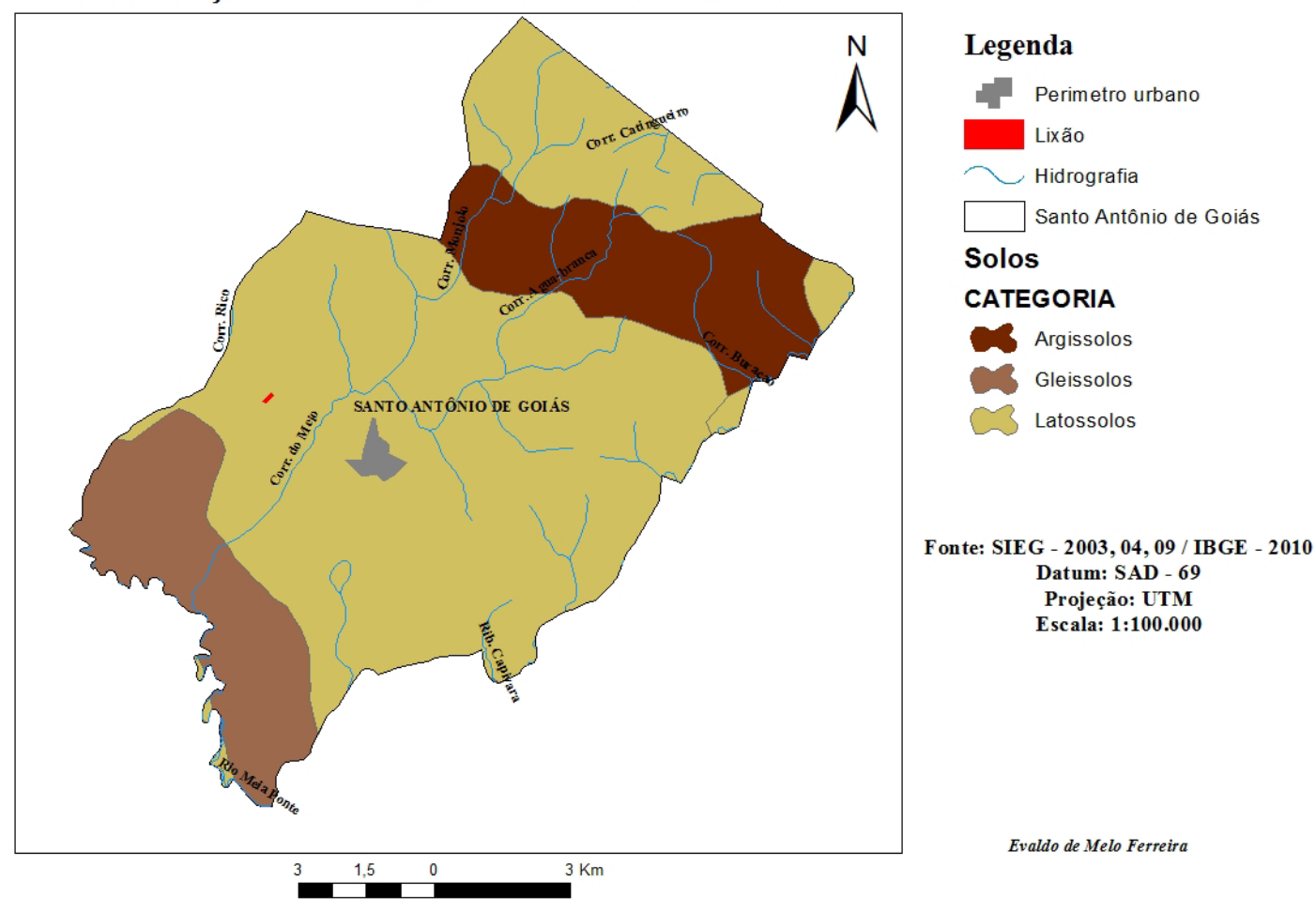

Figura 4 - Espacialização da área de disposição final dos resíduos de Santo Antônio de Goiás (em vermelho) em relação ao centro urbano e ao Córrego do Meio.

\section{5. Índice de Qualidade de Aterro de Resíduos (IQR)}

A avaliação da área de disposição utilizando o IQR constituiu-se de três partes distintas relacionadas a aspectos locacionais, estruturais e operacionais. Os resultados para a área de disposição de resíduos do município de Santo Antônio de Goiás são mostrados nos Quadros 2, 3 e 4. 
Quadro 2 - Características locais da área de disposição do município de Santo Antônio de Goiás.

\begin{tabular}{|c|c|c|c|c|}
\hline ÍTEM & SUB-ÍTEM & AVALIAÇÃO & PESO & PONTOS \\
\hline & \multirow{2}{*}{$\begin{array}{l}\text { CAPACIDADE DE SUPORTE } \\
\text { DO SOLO }\end{array}$} & ADEQUADA & 5 & 5 \\
\hline & & INADEQUADA & 0 & \\
\hline & \multirow{2}{*}{$\begin{array}{l}\text { PROXIMIDADE DE NÚCLEOS } \\
\text { HABITACIONAIS }\end{array}$} & LONGE $>500 \mathrm{~m}$ & 5 & 5 \\
\hline \multirow{2}{*}{$\begin{array}{l}\mathbf{C} \\
\mathbf{A} \\
\mathbf{R}\end{array}$} & & PRÓXIMO & 0 & \\
\hline & \multirow{2}{*}{$\begin{array}{l}\text { PROXIMIDADE DE CORPOS } \\
\text { DÁGUA }\end{array}$} & LONGE $>200 \mathrm{~m}$ & 3 & 3 \\
\hline \multirow{3}{*}{$\begin{array}{l}\mathbf{A} \\
\mathbf{C} \\
\mathbf{T}\end{array}$} & & PRÓXIMO & 0 & \\
\hline & \multirow{3}{*}{$\begin{array}{l}\text { PROFUNDIDADE DO LENÇOL } \\
\text { FREÁTICO }\end{array}$} & MAIOR 3m & 4 & NA \\
\hline & & DE 1 a $3 \mathrm{~m}$ & 2 & NA \\
\hline \multirow{3}{*}{$\begin{array}{l}\mathbf{E} \\
\mathbf{R}\end{array}$} & & DE 0 a $3 \mathrm{~m}$ & 0 & NA \\
\hline & \multirow{3}{*}{ PERMEABILIDADE DO SOLO } & BAIXA & 5 & NA \\
\hline & & MÉDIA & 2 & NA \\
\hline \multirow{2}{*}{$\begin{array}{l}\mathbf{S} \\
\mathbf{T} \\
\mathbf{I}\end{array}$} & & ALTA & 0 & NA \\
\hline & \multirow{3}{*}{$\begin{array}{l}\text { DISPONIBILIDADE DE } \\
\text { MATERIAL PARA } \\
\text { RECOBRIMENTO }\end{array}$} & SUFICIENTE & 4 & \\
\hline C & & INSUFICIENTE & 2 & 2 \\
\hline \multirow{3}{*}{$\begin{array}{l}\text { A } \\
\text { S }\end{array}$} & & NENHUMA & 0 & \\
\hline & \multirow{2}{*}{$\begin{array}{l}\text { QUALIDADE DO MATERIAL } \\
\text { PARA RECOBRIMENTO }\end{array}$} & BOA & 2 & 2 \\
\hline & & RUIM & 0 & \\
\hline \multirow{2}{*}{$\begin{array}{l}\text { D } \\
\text { o }\end{array}$} & \multirow{3}{*}{$\begin{array}{l}\text { CONDIÇÕES DE SISTEMA } \\
\text { VIÁRIO, TRÂNSITO E ACESSO }\end{array}$} & BOAS & 3 & \\
\hline & & IRREGULARES & 2 & \\
\hline \multirow{6}{*}{$\begin{array}{l}\mathbf{L} \\
\mathbf{O} \\
\mathbf{C} \\
\mathbf{A} \\
\mathbf{L}\end{array}$} & & RUINS & 0 & 0 \\
\hline & \multirow{2}{*}{$\begin{array}{l}\text { ISOLAMENTO VISUAL DA } \\
\text { VIZINHANÇA }\end{array}$} & BOM & 4 & \\
\hline & & RUIM & 0 & 0 \\
\hline & \multirow{2}{*}{$\begin{array}{l}\text { LEGALIDADE DE } \\
\text { LOCALIZAÇÃO }\end{array}$} & LOCAL PERMITIDO & 5 & 5 \\
\hline & & LOCAL PROIBIDO & 0 & \\
\hline & TOTAL & & & 22 \\
\hline & el), de & & & \\
\hline
\end{tabular}

Fonte: Adaptado de CETESB (2011)

Quadro 3 - Infraestrutura da área de disposição do município de Santo Antônio de Goiás.

\begin{tabular}{|c|c|c|c|c|}
\hline ÍTEM & SUB-ÍTEM & AVALIAÇÃ̃O & PESO & PONTOS \\
\hline & \multirow{2}{*}{ CERCAMENTO DA ÁREA } & SIM & 2 & 2 \\
\hline & & NÃO & 0 & \\
\hline & \multirow{2}{*}{ PORTARIA / GUARITA } & SIM & 2 & \\
\hline & & NÃO & 0 & 0 \\
\hline & \multirow{2}{*}{$\begin{array}{l}\text { IMPERMEABILIZAÇÃO DA } \\
\text { BASE DO ATERRO }\end{array}$} & SIM / DESNECES. & 5 & \\
\hline & & \begin{tabular}{|l|} 
NÃO \\
\end{tabular} & 0 & 0 \\
\hline & \multirow{3}{*}{ DRENAGEM DO CHORUME } & SUFICIENTE & 5 & \\
\hline & & INSUFICIENTE & 1 & \\
\hline & & INEXISTENTE & 0 & 0 \\
\hline & \multirow{3}{*}{$\begin{array}{l}\text { DRENAGEM DE ÁGUAS } \\
\text { PLUVIAIS DEFINITIVA }\end{array}$} & SUFICIENTE & 4 & \\
\hline & & INSUFICIENTE & 2 & \\
\hline & & INEXISTENTE & 0 & 0 \\
\hline & \multirow{3}{*}{$\begin{array}{l}\text { DRENAGEM DE ÁGUAS } \\
\text { PLUVIAIS PROVISÓRIA }\end{array}$} & SUFICIENTE & 2 & \\
\hline & & INSUFICIENTE & 1 & \\
\hline \multirow{4}{*}{$\begin{array}{l}\text { I } \\
\mathbf{N} \\
\mathbf{F} \\
\mathbf{R} \\
\mathbf{A}\end{array}$} & & INEXISTENTE & 0 & 0 \\
\hline & \multirow{3}{*}{$\begin{array}{l}\text { TRATOR DE ESTEIRAS OU } \\
\text { COMPATIVEL }\end{array}$} & PERMANENTE & 5 & 5 \\
\hline & & \begin{tabular}{|l} 
PERIODICAMENTE \\
\end{tabular} & 2 & \\
\hline & & INEXISTENTE & 0 & \\
\hline \multirow{2}{*}{$\begin{array}{l}\mathbf{E} \\
\mathbf{S}\end{array}$} & \multirow{2}{*}{ OUTROS EQUIPAMENTOS } & SIM & 1 & \\
\hline & & NÃO & 0 & 0 \\
\hline \multirow{18}{*}{$\begin{array}{l}\mathbf{T} \\
\mathbf{R} \\
\mathbf{U} \\
\mathbf{T} \\
\mathbf{U} \\
\mathbf{R} \\
\mathbf{A}\end{array}$} & \multirow{2}{*}{\begin{tabular}{|l} 
SISTEMA DE \\
TRATAMENTO DE \\
CHORUME \\
\end{tabular}} & SUFICIENTE & 5 & \\
\hline & & INSUF. / INEXIST. & 0 & 0 \\
\hline & \multirow{2}{*}{\begin{tabular}{|l} 
ACESSO À FRENTE DE \\
TRABLHO \\
\end{tabular}} & BOM & 3 & \\
\hline & & RUIM & 0 & 0 \\
\hline & \multirow{2}{*}{ VIGILANTES } & SIM & 1 & \\
\hline & & NÃO & 0 & 0 \\
\hline & \multirow{3}{*}{$\begin{array}{l}\text { SISTEMA DE DRENAGEM } \\
\text { DE GASES }\end{array}$} & SUFICIENTE & 3 & \\
\hline & & \begin{tabular}{|l|} 
INSUFICIENTE \\
\end{tabular} & 1 & \\
\hline & & INEXISTENTE & 0 & 0 \\
\hline & \multirow{2}{*}{$\begin{array}{l}\text { CONTROLE RECEBIMENTO } \\
\text { DE CARGAS }\end{array}$} & SIM & 2 & \\
\hline & & \begin{tabular}{|l|} 
NÃO \\
\end{tabular} & 0 & 0 \\
\hline & \multirow{3}{*}{$\begin{array}{l}\text { MONITORAÇ̃̃O DE ÁGUAS } \\
\text { SUBTERRÂNEAS }\end{array}$} & SUFICIENTE & 3 & \\
\hline & & INSUFICIENTE & 2 & \\
\hline & & INEXISTENTE & 0 & 0 \\
\hline & \multirow{3}{*}{$\begin{array}{l}\text { ATENDIMENTO A } \\
\text { ESTIPILAÇÕES DE PROJETO }\end{array}$} & SIM & 2 & \\
\hline & & PARCIALMENTE & 1 & \\
\hline & & NÃO & 0 & 0 \\
\hline & TOTAL & & & 7 \\
\hline
\end{tabular}

Fonte: CETESB (2011). 
Quadro 4 - Condições Operacionais da área de disposição do município de Santo Antônio de Goiás.

\begin{tabular}{|c|c|c|c|c|}
\hline Í́TEM & SUB-ÍTEM & AVALIAÇÃ̃O & PESO & PONTOS \\
\hline \multirow[b]{11}{*}{3} & \multirow{2}{*}{ ASPECTO GERAL } & BOM & 4 & \\
\hline & & RUIM & 0 & 0 \\
\hline & \multirow{2}{*}{$\begin{array}{l}\text { OCORRÊNCIA DE LIXO A } \\
\text { DESCOBERTO } \\
\end{array}$} & NÃO & 4 & \\
\hline & & SIM & 0 & 0 \\
\hline & \multirow{3}{*}{ RECOBRIMENTO DO LIXO } & ADEQUADO & 4 & \\
\hline & & INADEQUADO & 1 & 1 \\
\hline & & INEXISTENTE & 0 & \\
\hline & \multirow{2}{*}{$\begin{array}{l}\text { PRESENCCA DE URUBUS OU } \\
\text { GAIVOTAS }\end{array}$} & NÃO & 1 & \\
\hline & & SIM & 0 & 0 \\
\hline & \multirow{2}{*}{$\begin{array}{l}\text { PRESENCCA DE MOSCAS EM } \\
\text { GRANDE QUANTIDADE }\end{array}$} & NÃO & 2 & \\
\hline & & SIM & 0 & 0 \\
\hline \multirow{2}{*}{$\begin{array}{l}\mathbf{C} \\
\mathbf{O}\end{array}$} & \multirow{2}{*}{ PRESENÇA DE CATADORES } & NÃO & 3 & \\
\hline & & SIM & 0 & 0 \\
\hline \multirow{7}{*}{$\begin{array}{l}\mathbf{N} \\
\mathbf{D} \\
\mathbf{I} \\
\mathbf{C} \\
\tilde{\mathbf{O}} \\
\mathbf{E} \\
\mathbf{S}\end{array}$} & \multirow{2}{*}{$\begin{array}{l}\text { CRIAÇÃO DE ANIMAIS } \\
\text { (PORCOS, BOIS) }\end{array}$} & NÃO & 3 & 3 \\
\hline & & SIM & 0 & \\
\hline & \multirow{2}{*}{$\begin{array}{l}\text { DESCARGA DE RESÍDUOS DE } \\
\text { SERVIÇO DE SAÚDE }\end{array}$} & NÃO & 3 & 3 \\
\hline & & SIM & 0 & \\
\hline & \multirow{2}{*}{\begin{tabular}{|l|} 
DESCARGA DE RESÍDUOS \\
INDUSTRIAIS \\
\end{tabular}} & NÃO / ADEQUADA & 4 & 4 \\
\hline & & SIM / INADEQUADA & 0 & \\
\hline & \multirow{3}{*}{$\begin{array}{l}\text { FUNCIONAMENTO DA } \\
\text { DRENAGEM PLUVIAL } \\
\text { DEFINITIVA } \\
\end{array}$} & BOM & 2 & \\
\hline \multirow{2}{*}{$\begin{array}{l}\mathbf{O} \\
\mathbf{P}\end{array}$} & & IRREGULAR & 1 & \\
\hline & & INEXISTENTE & 0 & 0 \\
\hline \multirow{6}{*}{$\begin{array}{l}\mathbf{E} \\
\mathbf{R} \\
\mathbf{A} \\
\mathbf{C} \\
\mathbf{I} \\
\mathbf{o} \\
\mathbf{N}\end{array}$} & \multirow{3}{*}{\begin{tabular}{|l} 
FUNCIONAMENTO DA \\
DRENAGEM PLUVIAL \\
PROVISÓRIA
\end{tabular}} & BOM & 2 & \\
\hline & & IRREGULAR & 1 & \\
\hline & & INEXISTENTE & 0 & 0 \\
\hline & \multirow{3}{*}{$\begin{array}{l}\text { FUNCIONAMENTO DA } \\
\text { DRENAGEM DE CHORUME }\end{array}$} & BOM & 3 & \\
\hline & & REGULAR & 2 & \\
\hline & & INEXISTENTE & 0 & 0 \\
\hline \multirow{12}{*}{$\begin{array}{l}\text { A } \\
\text { S }\end{array}$} & \multirow{3}{*}{$\begin{array}{l}\text { FUNCIONAMENTO DO } \\
\text { SISTEMA DE TRATAMENTO } \\
\text { DE CHORUME }\end{array}$} & BOM & 5 & \\
\hline & & REGULAR & 2 & \\
\hline & & INEXISTENTE & 0 & 0 \\
\hline & \multirow{3}{*}{\begin{tabular}{|l} 
FUNCIONAMENTO DO SIST. \\
DE MONITORAÇÃO DE \\
ÁGUAS SUBTERRÂNEAS
\end{tabular}} & BOM & 2 & \\
\hline & & REGULAR & 1 & \\
\hline & & INEXISTENTE & 0 & 0 \\
\hline & \multirow{2}{*}{$\begin{array}{l}\text { EFICIÊNCIA DA EQUIPE DE } \\
\text { VIGILÂNCIA }\end{array}$} & $\mathrm{BOA}$ & 1 & \\
\hline & & RUIM & 0 & 0 \\
\hline & \multirow{3}{*}{$\begin{array}{l}\text { MANUTENÇÃO DOS ACESSOS } \\
\text { INTERNOS }\end{array}$} & BOAS & 2 & \\
\hline & & REGULARES & 1 & \\
\hline & & PÉSSIMAS & 0 & 0 \\
\hline & TOTAL & & & 11 \\
\hline
\end{tabular}

Fonte: CETESB (2011).

Por meio dos três subitens respondidos foi possível calcular o IQR da área de estudo (Equação 1). O IQR obtido foi 3,07, mostrando que as condições da área estão inadequadas. Uma vez que o valor se encontra menor que 6,1.

Equação 1. IQR = (Sub1 + Sub 2 + Sub 3) $/ 13$

Onde, Sub1 é o subtotal do primeiro quadro; Sub2 é o subtotal do segundo quadro e Sub 3 é o subtotal do terceiro quadro. Logo:

$$
\begin{aligned}
& \mathrm{IQR}=22+7+11 / 13 \\
& \mathrm{IQR}=3,07
\end{aligned}
$$

\section{CONCLUSÕES}

O município de Santo Antônio de Goiás mesmo tendo possuído licença ambiental, está com a disposição final de resíduos sólidos em total desacordo com a legislação brasileira. Após a avaliação 
da área por meio do método IQR, os resultados obtidos não foram bons, comprovando que a área está inadequada. Foi identificada a necessidade de reavaliar a composição gravimétrica dos resíduos gerados no município, utilizando de método mais apurado.

Santo Antônio de Goiás fez no ano de 2013 o PGIRS e existe a proposta para formação de consórcio entre Inhumas, Brazabrantes, Goianira e Nova Veneza. Caso isso não ocorra e a área de disposição atual continue sendo utilizada, é necessária a adoção de medidas técnicas/tecnológicas para a conversão da área em aterro sanitário simplificado, necessitando para isto da aquisição de uma nova área.

Deve-se fazer a impermeabilização da nova vala, construção de sistema para a drenagem do percolado líquido e dos gases, construção de lagoa para recebimento e tratamento do chorume, construção de sistema para captação do material tratado, análise da capacidade de autodepuração e também resiliência do corpo receptor, remoção de catadores e animais, construção de vias para o trânsito de veículos, estabelecimento de uma nova cerca viva, galpão coberto para recebimento dos recicláveis, construção de sede administrativa e balança para pesagem dos caminhões que chegam com os resíduos (medidas que já deviam ter sido adotadas de acordo com a Lei 12.305, até no máximo agosto de 2012).

\section{REFERÊNCIAS}

ABES - ASSOCIAÇÃO BRASILEIRA DE ENGENHARIA SANITÁRIA E AMBIENTAL. Municípios mineiros se unem para acabar com os lixões. Disponível em: <http://www.abes-mg.org.br/visualizacao-declippings/pt-br/ler/1705/municipios-mineiros-se-unem-para-acabar-com-os-lixoes>. Acesso em: 20 fev. 2014.

\section{ABRELPE - ASSOCIAÇÃO BRASILEIRA DE EMPRESAS DE LIMPEZA PUBLICA E RESÍDUOS ESPECIAIS. Panorama dos resíduos sólidos no Brasil-2012. São Paulo: ABRELPE, 2012. 116 p.}

ANVISA - Agência Nacional de Vigilância Sanitária. Resolução RDC No 306, de 7 de dezembro de 2004. Disponível em: <http://bvsms.saude.gov.br/bvs/saudelegis/anvisa/2004/res0306_07_12_2004.html>. Acesso em: 26 fev. 2013.

ABNT - ASSOCIAÇÃO BRASILEIRA DE NORMAS TÉCNICAS (ABNT). NBR 8419: Apresentação de projetos de aterros sanitários de resíduos sólidos urbanos. Rio de Janeiro, 1992.

NBR 13896: Aterros de resíduos não perigosos-Critérios para projeto, implantação e operação. Rio de Janeiro, 1997.

BRASIL. Lei $\mathrm{N}^{\circ}$ 12.305, de 2 de agosto de 2010. Institui a Política Nacional de Resíduos Sólidos; altera a Lei no 9.605, de 12 de fevereiro de 1998; e dá outras providências. Disponível em: <http://www.planalto.gov.br/ ccivil_03/_ato2007-2010/2010/lei/112305.htm>. Acesso em: 02 jan. 2014.

CETESB - COMPANHIA ESTADUAL DE SÃO PAULO. Inventário Estadual de Resíduos Sólidos Urbanos, 2011. 21 ed. São Paulo: CETESB, 2011. 110 p.

Resíduos urbanos, de serviços de saúde e da construção civil. Disponível em: <http://www.cetesb. sp.gov.br/residuos-solidos/residuos-urbanos/2-residuos-urbanos>. Acesso em: 01 mar. 2014.

CNM - Confederação Nacional dos Municípios. Resíduos Sólidos: Nova Demanda. Disponível em: <http:// geografia.uol.com.br/geografia/mapas-demografia/50/residuos-solidos-novas-demandas-de-acordo-com-aconfederacao-293243-1.asp.>. Acesso em: 27 fev. 2014.

CONAMA - Conselho Nacional do Meio Ambiente. Resolução N 307, de 05 de julho de 2002. Estabelece diretrizes, critérios e procedimentos para a gestão dos resíduos da construção civil. Disponível em: <http:// www.mma.gov.br/port/conama/legiabre.cfm?codlegi=307>. Acesso em: 02 jan. 2014. 
Resolução $\mathrm{N}^{\circ}$ 358, de 29 de abril de 2005. Dispõe sobre o tratamento e a disposição final dos resíduos dos serviços de saúde e dá outras providências. Disponível em: <http://www.mma.gov.br/port/conama/res/ res05/res35805.pdf>. Acesso em: 02 jan. 2014.

Resolução N$^{\circ} 431$, de 24 de maio de 2011. Altera o art. $3^{\circ}$ da Resolução no 307, de 5 de julho de 2002, do Conselho Nacional do Meio Ambiente CONAMA, estabelecendo nova classificação para o gesso. Disponível em: <http://www.mma.gov.br/port/conama/legiabre.cfm?codlegi=649>. Acesso em: 27 fev. 2014.

EMBRAPA - Empresa Brasileira de Pesquisa Agropecuária. Agência de Informação Embrapa, Bioma Cerrado, 2007. Latossolos. Disponível em: <http://www.agencia.cnptia.embrapa.br/Agencia16/AG01/arvore/ AG01_96_10112005101956.html>. Acesso em: 06 mar. 2014.

Sistema Brasileiro de Classificação de Solos. 2 ed. Rio de Janeiro: EMBRAPA-SPI, 2006. 306 p.

GOIÁS. SEMARH - SECRETARIA DO MEIO AMBIENTE E DOS RECURSOS HÍDRICOS DO

ESTADO DE GOIÁS. Relatório de Vistoria na Área de Disposição Final dos Resíduos Sólidos Urbanos do Município de Santo Antônio de Goiás, 2011.

Instrução Normativa no 11 de 2013-Aterro Sanitário Simplificado. Disponível em: <http:// www.semarh.goias.gov.br/site/uploads/files/legislacao_semarh/instrucoes_normativas/instruins_normativa_n_011-2013.pdf>. Acesso em 05 fev. 2014.

IBGE - Instituto Brasileiro de Geografia e Estatística. Goiás-Santo Antônio de Goiás-infográficos: dados gerais do município. Disponível em: <http://www.cidades.ibge.gov.br/painel/painel.php?lang=\&codmun $=521973 \&$ search=goias\%7Csanto-antonio-de-goias\%7Cinfograficos:-dados-gerais-do-municipio $>$. Acesso em: 20 dez. 2013.

IFWIS - Idaho Fish and Wildlife Information System (IFWIS). ArcMap 10.1 - Edit the Service Layer Credit. Disponível em: <http://fishandgame.idaho.gov/ifwis/portal/taxonomy/term/453>. Acesso em: 06 mar. 2014.

JACOBI, P. R \& BESSEN, G. R. Gestão de resíduos sólidos em São Paulo: desafios da sustentabilidade. Estudos Avançados, São Paulo, vol. 25, n. 71, p. 135-158, jan./abr. 2011.

LIMA, A. S \& CABRAL, A. E. B. Caracterização e classificação dos resíduos de

construção civil da cidade de Fortaleza (CE). Engenharia Sanitária e Ambiental, Rio de Janeiro, vol. 18, n. 2, p. 169-176, abr./jun. 2013.

MMA-MINISTÉRIO DO MEIO AMBIENTE. Inclusão social de catadores. Disponível em: <http:// www.mma.gov.br/responsabilidade-socioambiental/a3p/eixos-tematicos/gestão-adequada-dos-resíduos/ item/9341>. Acesso em: 20 fev. 2014.

SANTO ANTÔNIO DE GOIÁS. Plano municipal de gestão integrada de resíduos sólidos. Santo Antônio de Goiás: Innove Consultoria Ambiental, 2013. 80 p.

SIEG - Sistema Estadual de Geoinformação de Goiás. Download de Arquivos SIG (Shapefile). Base de dados de 2004. Disponível em: <http://www.sieg.go.gov.br/>. Acesso em: 06 mar. 2014.

SIMONETTO, E. O \& LÖBLER, M. L. Simulação baseada em system dynamics para avaliação de cenários sobre geração e disposição de resíduos sólidos urbanos. Produção, Santa Maria, vol.-, n.-, p. 0-0, ahead of print Epub, jul. 2013. 